\title{
Guest Editorial for the Special Section on "Algorithm Vs. Architectures: Opportunities and Challenges in Multicore/GPU DSP
}

\author{
Gwo Giun Chris Lee ${ }^{1,2} \cdot$ Scott C. Kim ${ }^{3}$
}

Published online: 14 October 2017

(C) Springer Science+Business Media, LLC 2017

Based upon mathematical fundamentals as foundations for low-complexity algorithms, intelligent, flexible, and efficient, analytics architectures including both software and hardware for GPU, multicore, high performance computing, and distributed systems, the current special section innovates papers on multicore/GPU DSP, in expediting the field of signal and information processing systems into futuristic new era based on Algorithm/Architecture Co-Exploration.

This special section in Journal of Signal Processing features opportunities and challenges in Multicore/GPU DSP based on the exploration of both algorithm and Architecture and consists of five papers with brief summary from the authors as stipulated below.

The first paper, "Parallel Digital Predistortion Design on Mobile GPU and Embedded Multicore CPU for Mobile Transmitters" (https://doi.org/10.1007/s11265-017-1233-y) by Li et al., present high data rate implementations of broadband DPD on modern embedded processors, such as mobile GPU and multicore CPU, by taking advantage of emerging parallel computing techniques for exploiting their computing resources.

The second paper, "Complexity-Aware Gabor Filter Bank Architecture Using Principal Component Analysis" (https://doi. org/10.1007/s11265-017-1246-6) by Chen et al., proposed a computationally efficient method for Gabor filter bank through Principal Component Analysis (PCA) analysis and low rank

Gwo Giun Chris Lee

gwoglee@gmail.com

1 Department of Electrical Engineering, National Cheng Kung University, Tainan, Taiwan

2 Bioinfotronics Research Center, National Cheng Kung University, Tainan, Taiwan

3 University of Maryland, College Park, MD, USA approximation based on algorithm/Architecture Co-Exploration (AAC).

The third paper, "Implementation of a Multirate Resampler for Multi-carrier Systems on GPUs" (https://doi.org/10.1007/ s11265-017-1239-5) by Kim et al., presented an all-softwarebased, fully parallel, multirate resampling method based on graphics processing units (GPUs). The proposed approach is well-suited for wireless communication systems that have simultaneous requirements on high throughput and low latency.

In the fourth paper, "A Hybrid Task Graph Scheduler for High Performance Image Processing Workflows" (https://doi.org/10.1007/s11265-017-1262-6) by Blattner et al., proposed a Hybrid Task Graph Scheduler (HTGS) which improves programmer productivity when implementing hybrid workflows for multi-core and multiGPU systems. The Hybrid Task Graph Scheduler (HTGS) is an abstract execution model, framework, and API that increases programmer productivity when implementing hybrid workflows for such systems.

In the fifth and final paper, "Design flow for GPU and multicore execution of dynamic dataflow programs" (https://doi.org/10.1007/s11265-017-1260-8) by Boutellier et al., proposes a novel design flow for mapping RVC-CAL dataflow programs to parallel and heterogeneous execution platforms. Through the proposed design flow the programmer can describe an application in the RVC-CAL language and map it to multi- and manycore platforms, as well as GPUs, for efficient execution. The functionality and efficiency of the proposed approach is demonstrated by a parallel implementation of a video processing application and a run-time reconfigurable filter for telecommunications.

The guest editors sincerely acknowledge the EiC of this journal for the kind support, the authors for the interesting papers so contributed, and the reviewers for the 
high-quality review and feedbacks in making the content of the papers even better.

We hope you will enjoy this special section with heuristic ideas in signal processing systems!

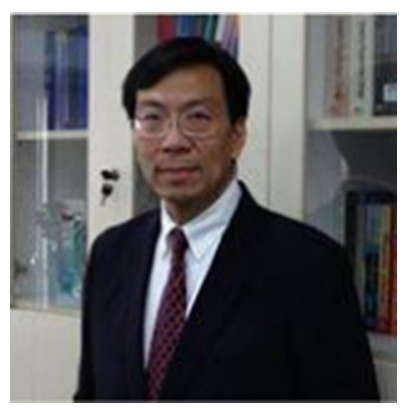

Gwo Giun Chris Lee is an investigator in the field of Data Science including multimedia and bioinformatics. His endeavors in system design, based on analytics of algorithm concurrently with architecture, has made possible computations on System-on-Chip and cloud platforms in resolving complex problems with both accuracy and efficiency. Having previously held leading and managerial positions in the industry such as System Architect in former Philips Semiconductor in Silicon Valley, Lee was recruited to NCKU in 2003 where he found and is currently directing the Bioinfotronics Research Center (BITS). BITS conducts highly multidisciplinary research having collaborations with IBM TJ Watson Research Center on Cloud Computing; Inform Genomics Inc., found by experts from MD Anderson Cancer Research Center, Harvard Medical School, and MIT, on analytics architecture for Precision Medicine; Banner Health Research on Intelligent Health Cloud for Alzheimer Disease; National Taiwan University on Harmonically Generated Microscopy Medical Image Processing; etc. Representing Taiwan and US in ITU and MPEG of ISO/IEC, Lee led the international standardization efforts for video coding from several aspects. His invention of the novel Algorithm/ Architecture Co-exploration system design methodology was substantial in guiding world experts in delivering feasible and realistic standards for applications requiring processing of big multimedia data. His lowcomplexity 3D video coding technology was also adopted by MPEG. Lee received his B.S. degree in electrical engineering from National Taiwan University and both his M.S. and Ph.D. degrees in electrical engineering from University of Massachusetts. He has contributed more than 130 original research and technical publications with the invention of $100+$ patents worldwide. Lee serves as the AE for IEEE TSP. He was formerly the AE for IEEE TCSVT for which he received the Best Associate Editor's Award in 2011. He has also served as the AE for JSPS since 2010.

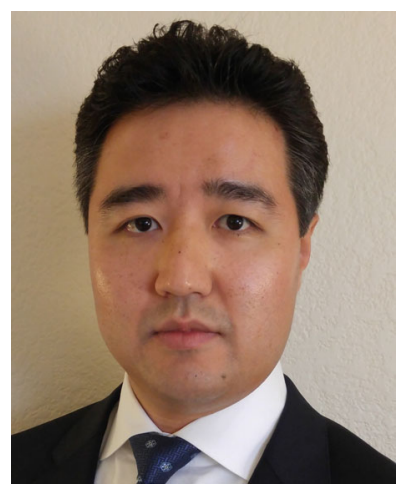

Scott C. Kim has been working in the electronics industry since 2000 , and he specializes in the design and implementation of signal processing systems (DISPS). His expertise also covers signal processing for wireless communication systems and embedded devices, such as FPGAs, GPUs, and SoCs. His research interests include parallel programming for heterogeneous computing and baseband radio modem design. He received the B.S. degree from the University of Michigan at Ann Arbor, the M.S. degree from Johns Hopkins University, and the Ph.D. degree from the University of Maryland at College Park. All of his degrees are in Electrical and Computer Engineering. He is a Senior Member of the IEEE. 